\title{
Propuesta didáctica para la enseñanza de los números enteros ${ }^{1}$
}

Didactic proposal for teaching integers

Proposta didática para o ensino de números inteiros

Recibido: mayo 2013

Aceptado: agosto 2013
Jorge Alejandro Rojas Gómez ${ }^{2}$

Aura Alejandra Ariza Daza ${ }^{3}$

\section{Resumen}

Este artículo pretende evidenciar la importancia que tiene la enseñanza de los números enteros, siendo este un proceso de bastante complejidad, el cual debe buscar por diversos medios generar un aprendizaje significativo en los estudiantes, para esto escogimos como metodología la teoría de situaciones didácticas de Brousseau, siendo esta una herramienta que permitirá a través de unas actividades, la construcción del objeto matemático por parte de los estudiantes, para determinar este aprendizaje se realizará una evaluación constante en los estudiantes con el fin de observar y analizar que ocurre en los diversos aprendizajes individuales, llegando de ese modo a una reflexión sobre lo ocurrido durante toda la gestión del docente.

Palabras clave: Matemáticas escolares; números; estructuras numéricas; números enteros; teoría de situaciones didácticas; enseñanza; planificación del profesor; metodología de enseñanza.

\begin{abstract}
This article aims to show the importance of teaching integers, this being a fairly complex process, which should seek in various ways to generate meaningful learning in students chose as a methodology for this theory of didactic situations of Brousseau, this being a tool that will allow through some activities, the construction of the mathematical object on the part of students, to determine this learning will be an ongoing assessment on students in order to observe and analyze what happens in the various individual learning, thereby reaching a reflection on what happened during the entire teaching management.
\end{abstract}

Keywords: school Mathematics, numbers, numerical structures; integers; theory of didactic situations, teaching, teacher planning, teaching methodology.

1 Artículo de Investigación.

2 Universidad Distrital Francisco José de Caldas. Bogotá, Colombia. Contacto: alorojas10@hotmail.es

3 Universidad Distrital Francisco José de Caldas. Bogotá, Colombia. Contacto: aleja9312@gmail.com. 


\section{Resumo}

Este artigo tem como objetivo mostrar a importância de inteiros de ensino, sendo este um processo bastante complexo, que deve procurar em várias maneiras de gerar aprendizagem significativa nos alunos escolheram como uma metodologia para esta teoria das situações didáticas de Brousseau, sendo esta uma ferramenta que vai permitir que através de algumas atividades, a construção do objeto matemático por parte dos alunos, para determinar esse aprendizado será uma avaliação permanente sobre os alunos, a fim de observar e analisar o que acontece nos vários aprendizagem individual, atingindo, assim, uma reflexão sobre o que aconteceu durante a gestão de ensino inteiro.

Palavras-chave: Matemática da escola, números, estruturas numéricas; inteiros; teoria das situações didáticas, ensino, planejamento de professores, metodologia de ensino.

\section{Contextualización}

La presente experiencia de aula tienen lugar en el Colegio José Félix Restrepo sede A, donde se nos encomendó el diseño de una propuesta para la enseñanza del número entero en estudiantes de grado séptimo, esta propuesta se diseñó a través de la teoría de situaciones didácticas de Brousseau; para el diseño de esta se tuvo presente el crear una situación que lleve a los niños a construir los números enteros a partir de una necesidad generada en un medio, potenciando en ellos la visión del objeto matemático como algo útil y necesario, que se necesita para la vida diaria.

\section{Marco teórico}

Desde los inicios históricos de la Matemática podemos encontrar reflexiones acerca de las dificultades que pueden generar los números enteros (esencialmente números negativos) para su comprensión y total aceptación. El reconocimiento y la legitimación de este campo numérico sufrió durante un largo proceso, requiriendo de mucho tiempo para que los matemáticos reconocieran, aceptaran y legitimaran los números negativos, por lo que no debería resultar tan extraño que los alumnos presenten dificultades a la hora de construir conocimientos en torno a ellos.

Según Glaeser (1981) en la evolución histórica de la noción de número negativo desde sus primeras emergencias hasta el concepto actual, se pueden constatar los siguientes obstáculos:

Falta de aptitud para manipular cantidades negativas aisladas. Indica que la necesidad de efectuar cálculos algebraicos con diferencias y, en particular, la necesidad de multiplicar dos diferencias, le lleva a enunciar la regla de los signos y, sin embargo, no acepta la existencia de números negativos aislados.

Dificultad para dar sentido a las cantidades negativas aisladas. Conciben la existencia de soluciones negativas de las ecuaciones, las "ven" y las tienen en cuenta, pero no pueden aceptarlas como cantidades reales y las justifican diciendo, por ejemplo, que son cantidades ficticias que expresan un defecto en el enunciado del problema.

Dificultad para unificar la recta real. En el intento de sobrepasar el obstáculo anterior interpretando las cantidades negativas como cantidades reales, concibiendo los negativos y los positivos en términos antinómicos: "lo negativo" neutralizaba, se oponía a "lo positivo", pero era de naturaleza distinta. Es decir, la cantidad negativa era tan real como la positiva, pero estaba tomada en un sentido opuesto. Esta heterogeneidad que se establecía entre negativos y positivos no facilitaba su unificación en una única recta numérica y, en cambio, favorecía el modelo de dos semirrectas opuestas funcionando separadamente.

La ambigüedad de los dos ceros. Dificultad para pasar de un cero absoluto, un cero que significaba la ausencia de cantidad de magnitud, a un cero origen elegido arbitrariamente. Uno de los razonamientos 
más extendidos entre los matemáticos que se oponían a la consideración de las cantidades negativas como cantidades reales y no como meros artificios del cálculo, era que no se podía admitir la existencia de cantidades que fueran "menos que nada"

El estancamiento en el estadio de las operaciones concretas. La superación de los obstáculos anteriores permite aceptar los números negativos como cantidades reales y justificar su estructura aditiva, pero no así la estructura multiplicativa.( Cid, s.f, p.22-23).

Según Godino (2003), los números enteros se pueden introducir a través de transformaciones, movimientos y comparaciones con números naturales, permitiendo esto el paso a los números enteros $\mathrm{y}$ además un tratamiento que anticipará a la estructura aditiva de estos.

La metodología que se usara será basada en la teoría de situaciones didácticas (TSD) de Brousseau la cual corresponde a "un modelo de interacción de un sujeto con cierto medio que determina a un conocimiento dado como el recurso del que dispone el sujeto para alcanzar o conservar en este medio un estado favorable. Algunas de estas "situaciones" requieren de la adquisición 'anterior ' de todos los conocimientos y esquemas necesarios, pero hay otras que ofrecen una posibilidad al sujeto para construir por sí mismo un conocimiento nuevo en un proceso "genético".

El profesor ya ha planeado la situación didáctica de modo que existan momentos en que los alumnos interactúan con el problema, presenten conflictos cognitivos, se propicie la discusión y el debate y también hagan preguntas, Brousseau clasifica las situaciones didácticas en distintas fases para la construcción de un conocimiento, estas son:

\section{Descripción general}

La experiencia de aula que se quiere compartir tiene lugar en la ciudad de Bogotá y específicamente en el grado séptimo del colegio José Félix Restrepo (sede A). Inicialmente se hizo la aplicación de una prueba diagnóstico, la cual permitió identificar si los estudiantes resuelven problemas cuya estrategia de solución requiera el manejo de situaciones aditivas y multiplicativas en los números naturales, además si reconocen las propiedades de las operaciones en este mismo conjunto.

De acuerdo a los resultados de esta prueba se logro evidenciar que los estudiantes reconocen un número racional como un número natural, al tomar solo en cuenta el numerador o el denominador de éste. A demás de que se les dificulta desarrollar problemas aditivos compuestos, ya que los problemas aditivos y multiplicativos simples logran desarrollarlo con gran rapidez. Igualmente se observó que los estudiantes reconocen cuando una propiedad es verdadera en los números naturales pero no logran demostrarla por medio de un ejemplo. Teniendo en cuenta lo anterior se procede a generar esta propuesta la cual surge con el ideal de potenciar en los estudiantes la construcción de los números enteros, teniendo como metodología la teoría de situaciones didácticas.

La situación aplicada tenia como propósitos posibilitar la introducción a los números enteros a través de las transformaciones, potenciar en los estudiantes la noción de valor absoluto y valor relativo y viabilizar la diferenciación entre números naturales y enteros, en los estudiantes. En esta situación los estudiantes trabajaron en la fase de acción, formulación y validación, y los docentes realizan la institucionalización de los objetos matemáticos trabajados en ésta.

En la fase de acción se evidenció que los estudiantes tomaron todos los datos dados en la situación y los operaron, lo cual se debe a una translación directa del texto a la operación, en vez de crear una representación coherente del enunciado. Otros estudiantes hacen uso de representaciones pictóricas como ayuda para dar solución al problema, lo cual se puede tomar como un recurso manipulativo-grafico-textual (Godino, 1998, pág. 2). Pero no llega a reconocer que los números debajo del mar, los números con signo (-).

En las fases de formulación y validación varios grupos llegan a la repuesta correcta a partir de las transformaciones que se describían en la situación. Los estudiantes por medio de una representación 
pictórica logran identificar los números con signos, considerando la profundidad y la altura que representan indirectamente en la representación pictórica. Por lo que se puede deducir relacionando con lo expuesto por Vargas, Jinemo, \& Iriarte (1990. Págs.130-131) que el alumno descubre o intuye el comportamiento de los números por medio de un modelo intuitivo, en este caso real, en los que subyace parte de la aritmética de los números enteros. Introduciendo el número entero a partir de las profundidades y alturas.

Finalmente el docente realiza la institucionalización donde hace mención a partir de la situación de lo que es el valor absoluto y relativo; además de lo que es los números enteros y el reconocimiento de estos.

\section{Logros y dificultades}

Fase de acción:

- El estudiante toma lo datos que el problema daba y los opera, este error se puede relacionar con los que se presentan a la hora de resolver problemas,

- El estudiante hizo uso de una representación pictórica como ayuda para dar solución al problema, lo cual se puede tomar como un recurso manipulativo-grafico-textual.

- No resultó un problema fácil de resolver, por lo que es de esperar que los estudiantes tampoco puedan llegar de una vez por todas al concepto de número entero, pues es lo suficientemente complicado como para abarcarlo en una mirada

Fase de formulación y validación:

- Algunos estudiantes llegan a la solución del problema por medio de las operaciones realizadas, reconociendo los números con signo, creando un nuevo significado a los signos.

- Algunos estudiantes por medio de una representación pictórica logran identificar los números con signos, considerando la profundidad y la altura que representan indirectamente en la representación pictórica.

- Las dificultades de los alumnos para comprender y manipular correctamente los números positivos y negativos son, en cierta medida, un reflejo de las que históricamente tuvo la comunidad matemática para aceptarlos como números.

- Sin acordarse para nada de los negativos ni de lo que «saben» sobre ellos, respondan que no es posible o se queden perplejos sin contestar.

\section{Reflexión final}

El trabajar la TSD permite a los estudiantes potenciar cualquier aprendizaje de manera significativa, teniendo en cuenta las habilidades de cada persona, por ejemplo algunas personas desde el momento individual ya generan conocimiento, en cambio otros al trabajar en grupo o ver las exposiciones de sus compañeros, y por último los que aprenden simplemente con la explicación del docente, esto es lo más valioso que tiene trabajar la TSD, pues no hay un momento especifico en el cual se deba generar el aprendizaje, sino que puede ser en cualquier momento, quizás hasta por fuera del aula, cuando el estudiante reconozca un suceso con una estructura parecida a la de la situación, y tenga que enfrentarse a este de una manera nada escolar pero que si puede generar el conocimiento, de ahí que sugiera a los docentes que deseen aplicar esta situación el no desesperarse por ver un aprendizaje inmediato, sino que den lo mejor de cada uno y hagan conclusiones de este tipo hasta mínimamente finalizar la actividad.

\section{Referencias}

Brousseau, G. (1983).Obstáculos epistemológicos en la enseñanza de los números negativos. Zaratoga.

Cid, E. (s.f). La investigación didáctica sobre los números negativos: estado de la cuestión. Aragón: universidad de Zaragoza.

Glaeser, G. (1981), Epistemologie des nombres relatifs, Recherches en Didactique des Mathematiques, 2(3), 303-346.

Godino, J. (2003). Sistemas numéricos y su didáctica para maestros. Granada: Reprodigital. 